\title{
Surgical complications of liver resections
}

\author{
Sherif Abdel Halim, MD, El Sobky A. MD, Mohamed H Hamouda MD, \\ Youhanna Shohdy Shafik MD, MRCS(Eng), Mohamed E Seif, MD; \\ Moh A Marzouk MD
}

\author{
Department of General Surgery, Ain Shams University, Cairo, Egypt.
}

Background: Liver resection has been increasingly performed over the last 2 decades worldwide because of improved postoperative outcomes and evidence that this approach offers the only chance of cure in many patients.

Technical innovations have mainly focused on minimizing bleeding during transection of the hepatic parenchyma because excessive hemorrhage and the need for blood transfusion are associated with increased postoperative morbidity and mortality.

Liver resection is still a high risk procedure because of difficulties in preoperative assessment of functional liver volume and severity of co-morbidity, complexities of surgical procedures for preserving enough functional liver and preventing injury to major vessels in the remnant liver. Although zero mortality can be achieved during a limited period in some centers, the postoperative mortality in most studies is between $1 \%-5 \%$, even in patients with normal liver background.

Postoperative death can be attributed to liver failure, infection in surgical site, bleeding from esophageal varices, bile leakage, and extrahepatic diseases.

Methods: In this study, we classified postoperative complications into major and minor ones according to their fatal potential. We hypothesized that improved surgical technique can decrease surgical complications, while patient selection and careful perioperative management are important to prevent major complications. Randomized collection of twenty nine patients underwent partial hepatectomy for different indications. Analysis of every case has been done, retrospectively.

Results: In this study, the most common indication of liver resection was liver tumors accounting for $82.7 \%$ of cases, primary hepatic malignancy accounting for $75.8 \%$. Extension of liver resection was a predictor factor of complication. Major complications were observed with rate of $29.4 \%$ in patients who were subjected to major resections. The most common complication was liver decompensation accounting for $24.1 \%$, was observed in cirrhotic patients.

Conclusion: Our findings demonstrate that liver resection can be performed with low mortality and acceptable morbidity rates. Surgical complications can be reduced by employing meticulous surgical technique and, whenever indicated, vascular exclusion. In addition, use of vaccine against HBV eventually decreases the incidence, especially in endemic areas, and prevents the development of cirrhosis, which predisposes to hepatocellular carcinoma and liver decompensation after rersection.

\section{Introduction:}

Liver resections were first described centuries ago, but until the latter half of the 20th century, the majority of such resections were performed for management of either injuries or infections. ${ }^{1}$

Today, these procedures are performed not only for treatment of acute emergencies (e.g., traumatic injuries or abscesses) but also as potentially curative therapy for a variety 
of benign and malignant hepatic lesions. The most common malignant neoplasms (cancers) of the liver are metastasis; those arising from colorectal cancer are among the most common, and the most amenable to surgical resection. The most common primary malignant tumor of the liver is the hepatocellular carcinoma. ${ }^{2}$

Although hepatectomies have improved, post hepatectomy complications are taken in consideration; bleeding is the most feared technical complication and may be grounds for urgent reoperation. Biliary fistula is also a possible complication, one more amenable to non surgical management. Pulmonary complications such as atelectasis and pleural effusion are also common, and dangerous in patients with underlying lung disease. ${ }^{3}$

\section{Patients and methods:}

This study was carried in Ain Shams University Hospitals during the period from August 2010 to May 2012 including a randomized collection of twenty nine patients who underwent partial hepatectomy for different indications. Analysis of every case has been done, retrospectively.

Patients: A protocol was created in order to obtain the following data:

Age, sex, relevant past medical history, including cirrhosis, chronic hepatitis and previous neoplasm.

Indication of hepatectomy, intraoperative data such as tumor location, extension of resection, employment of vascular exclusion, blood transfusion requirements, information related to the pathologist report (tumor size and histological type).

Data regarding postoperative outcome, mainly postoperative complications, mortality index and hospital stay.

Methods: A bilateral subcostal incision was employed in all procedures, with an upward midline extension whenever necessary. Mobilization of liver was performed and cholecystectomy was indicated for cases of right hepatectomy. Vascular exclusion was done when necessary. Follow up was done at one, three, six months postoperatively.

Data were collected, coded, tabulated, and then analyzed using Microsoft $\AA$ office Excel 2003 computer software. Numerical variables were presented as mean and standard deviation while frequency and percent were used to present categorical variables.

\section{Results:}

A total of 29 liver resections were performed during the study period. The mean age was 48.2 years, ranging from 2 to 65 .

Twenty patients were males $(69 \%)$ and nine females (31\%).

In 16 patients $(55.2 \%)$ an underlying chronic liver disease was detected in the preoperative evaluation.

The most common indication was hepatic malignancy, which occurred in 24 patients $(82.7 \%)$. Primary hepatic malignancy was the indication for resection in 22 patients (75.8\%): Hepatocellular carcinoma in 18 $(62 \%)$, cholangiocarcinoma in $3(10.3 \%)$, and hepatoblastoma in $1(3.4 \%)$. Of the patients with hepatocellular carcinoma, 14 had liver cirrhosis.

Hepatic resection was performed for benign conditions in 5 patients, 2 with hemangioma, 1 with adenoma, 1 with inflammatory mass, 1 with traumatic rupture.

The type and extent of hepatic resections was determined according to site, size and extent of pathology. We had five patients who underwent right lobectomy, five underwent left extended hepatectomy, two for Left hepatectomy, seven had bisegmentectomy, and ten patients underwent segmentectomy.

Complications leading to life-threatening conditions were classified as MAJOR. These included major postoperative bleeding, any organic failure, intra-abdominal abscess, sepsis and portal vein thrombosis.

Complications with no fatal potential were considered MINOR and included pleural effusion, wound infection, urinary tract infection and atelectasis. Biliary leakage was also considered a minor complication since it was self-limited in the majority of cases.

Complications were observed in 17 patients $(58.6 \%)$. Major complications occurred in 5 patients $(17.2 \%)$. Hepatic insufficiency was the commonest major 

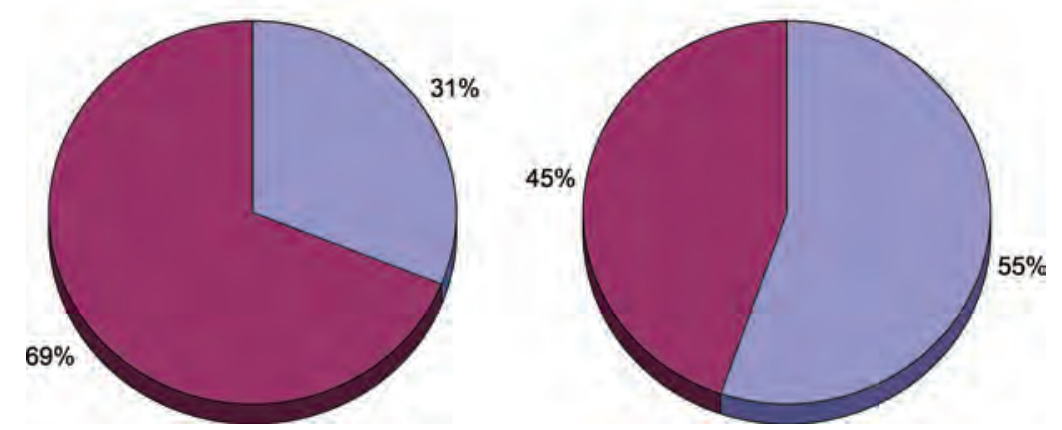

Figure (1): Showing preoperative liver condition.

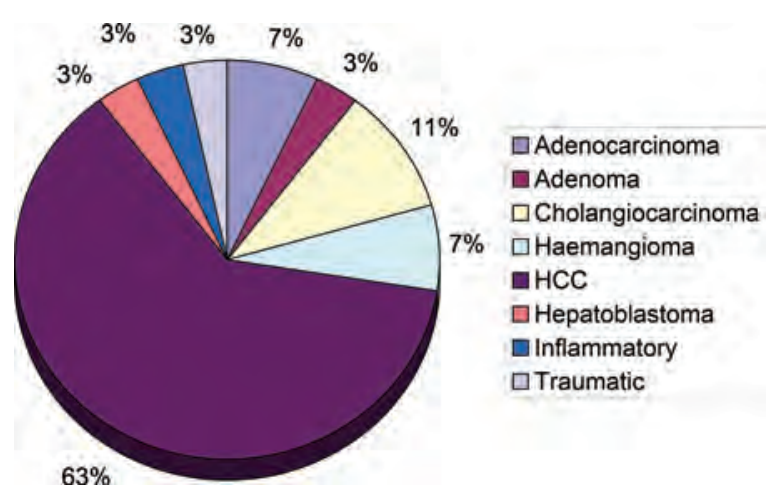

Figure (2): Showing indication of liver resection.

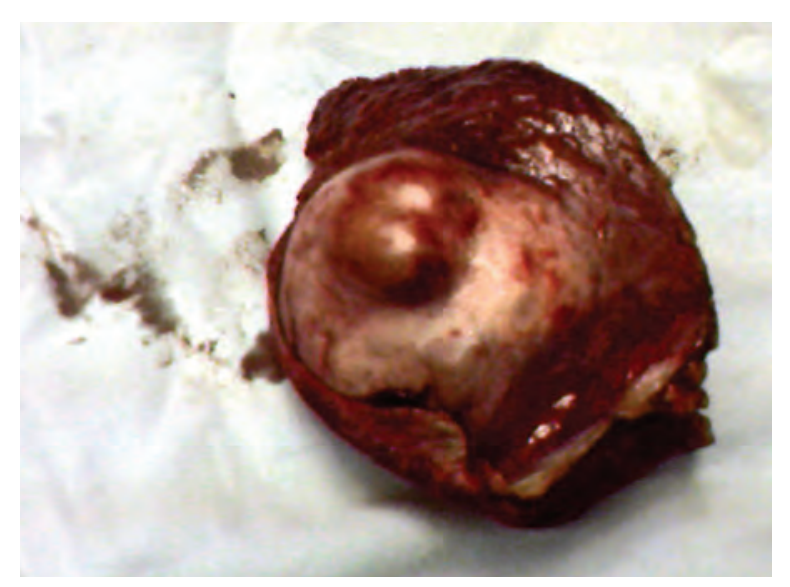

Figure (4): Left lateral segmentectomy showing tumor.

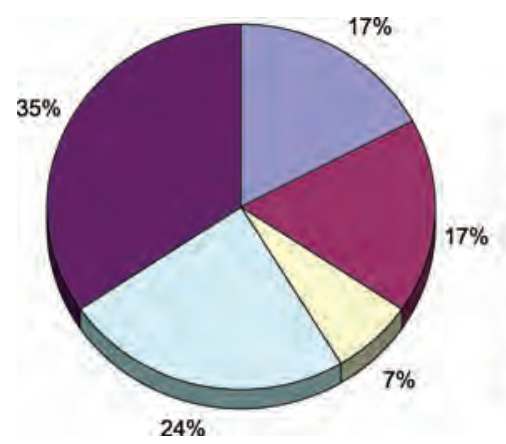

$\square$ Right lobectomy

- Left extended

hepatectomy

$\square$ Left hepatectomy

$\square$ Bisegmentectomy

- Segmentectomy

Figure (6): Showing extent of resection.

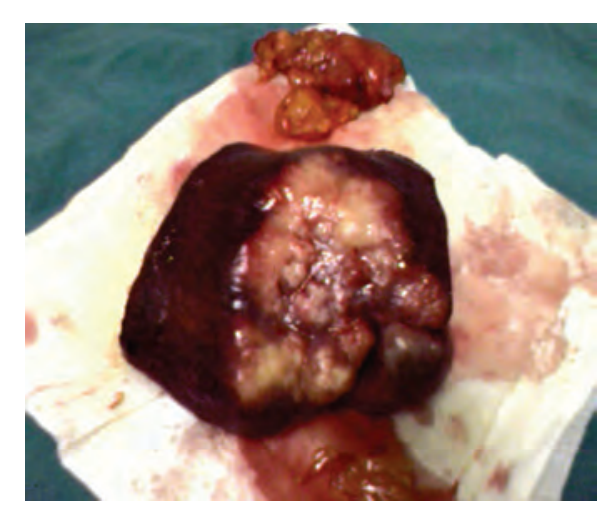

Figure (3): Right hepatectomy showing tumor.

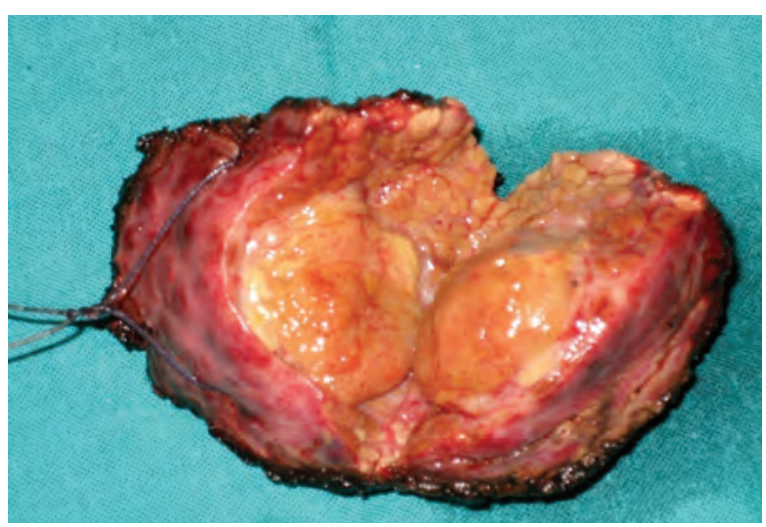

Figure (5): Segment VII resection

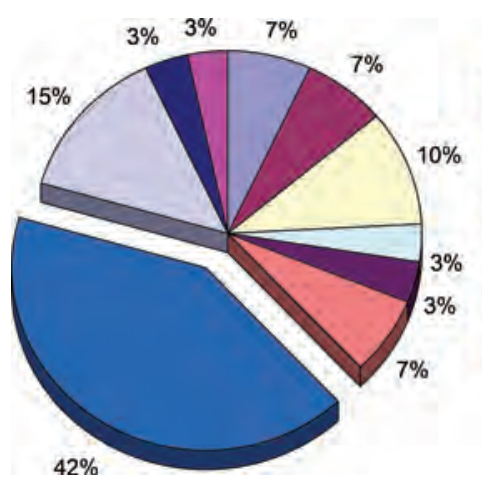

a Ascites \& chest infection - Ascites \& recurrence

$\square$ Ascites only

$\square$ Bleeding

Chest infection only

घIncisional hernia

No complication

$\square$ Recurrence only

- Renal failure

Wound infection

Figure (7): Showing range of complications. 
Table (1): Showing range of age.

\begin{tabular}{|l|l|l|l|l|}
\hline No & Mean & Std. Deviation & Minimum & Maximum \\
\hline 29 & 48.2 & 14.06 & 2 & 65 \\
\hline
\end{tabular}

Table (2): Showing sex differentiation.

\begin{tabular}{|l|l|l|}
\hline & Frequency & Percent \\
\hline Female & 9 & 31.0 \\
\hline Male & 20 & 69.0 \\
\hline Total & & 100.0 \\
\hline
\end{tabular}

Table (3): Showing preoperative liver condition.

\begin{tabular}{|l|l|l|}
\hline Liver condition & Frequency & Percent \\
\hline cirrhotic & 16 & 55.2 \\
\hline Non cirrhotic & 13 & 44.8 \\
\hline Total & 29 & 100.0 \\
\hline
\end{tabular}

Table (4): Showing indication of liver resection.

\begin{tabular}{|l|l|l|}
\hline Indication & Frequency & Percent \\
\hline Adenocarcinoma & 2 & 6.9 \\
\hline Adenoma & 1 & 3.4 \\
\hline Cholangiocarcinoma & 3 & 10.3 \\
\hline Haemangioma & 2 & 6.9 \\
\hline HCC & 18 & 62.1 \\
\hline Hepatoblastoma & 1 & 3.4 \\
\hline Inflammatory & 1 & 3.4 \\
\hline Traumatic & 1 & 3.4 \\
\hline Total & 29 & 100.0 \\
\hline
\end{tabular}

Table (6): Showing extent of resection.

\begin{tabular}{|l|l|l|}
\hline \multicolumn{1}{|c|}{ Procedure } & Frequency & Percent \\
\hline Right lobectomy & 5 & 17.2 \\
\hline Left extended hepatectomy & 5 & 17.2 \\
\hline Left hepatectomy & 2 & 6.8 \\
\hline Bisegmentectomy & 7 & 24.1 \\
\hline Segmentectomy & 10 & 34.4 \\
\hline
\end{tabular}

complication, occurring in seven patients (24.1\%). Recurrence developed in six cases $(20.6 \%)$, five of them underwent a major resection.

Intrabdominal bleeding occurred in 1 case (3.4\%). It was successfully managed with fluid infusion and blood transfusion.

Major complication rate varied with indication and extension of liver resection. These complications occurred in 2 of 3 patients (66.6\%) who underwent hepatectomy for cholangiocarcinoma, 10 of 18 patients 
Table 7: Showing range of complications.

\begin{tabular}{|l|l|l|}
\hline \multicolumn{1}{|c|}{ Complication } & Frequency & Percent \\
\hline Ascites \& chest infection & 2 & 6.9 \\
\hline Ascites \& recurrence & 2 & 6.9 \\
\hline Ascites only & 3 & 10.3 \\
\hline Bleeding & 1 & 3.4 \\
\hline Chest infection only & 1 & 3.4 \\
\hline Incisional hernia & 2 & 6.9 \\
\hline No complication & 12 & 41.4 \\
\hline Recurrence only & 4 & 13.8 \\
\hline Renal failure & 1 & 3.4 \\
\hline Wound infection & 1 & 3.4 \\
\hline Total & 29 & 100.0 \\
\hline
\end{tabular}

(55.5\%) for hepatocellular carcinoma, 2 of 2 patients $(100 \%)$ for metastatic disease, and 1 of 5 patients (20\%) for benign conditions. Major complications were observed in 5 of 17 patients $(29.4 \%)$ who were subjected to major resections.

\section{Discussion:}

Despite improvements in chemoembolization, cryosurgery and other modalities of treatment, liver resection remains the procedure of choice in the management of most primary liver tumors. Liver transplantation is the best approach in selected cases, mainly in patients with liver cirrhosis and small tumors. Hepatectomy is the mainstay treatment of selected patients with metastatic liver tumors, especially those in which the primary site is the large bowel. However, this procedure has been associated with significant morbidity and mortality. 4

In this study the most common indication of liver resection was liver tumors accounting for $82.7 \%$ of cases, primary hepatic malignancy accounting for $75.8 \%$, followed by benign conditions with $17.2 \%$ These results are compared to those of a recently published multicentric study reporting the outcomes of 2,097 patients subjected to hepatectomy. In their series, $52 \%$ of the patients underwent resection for metastatic disease and $16 \%$ for primary liver malignancy. ${ }^{5}$

Liver resection has been associated with high morbidity and mortality. The reported morbidity has ranged from $16.2 \%$ to $81 \%$. This enormous range is partially due to the heterogeneity of the published series, with major differences in indication, extension of hepatic resection, percentage of patients with cirrhosis and definition of postoperative complication. In this study, our rate of $58.6 \%$ is considered high when compared to others. However, we stress that a meticulous data review was employed so that complications such as pleural effusion were considered even when thoracocentesis and chest tube drainage were not required. Major complications occurred in $17.2 \%$ of patients and, analyzing specific complications, our rates are similar to those of major international centers. ${ }^{6}$

Risk factors for complications following hepatic resection have been addressed by others. Noguchi et al, detected albumin, glutamic oxaloacetate transaminase, serum total bilirubin, plasma disappearance rate of indocyanin green and $75 \mathrm{~g}$ oral glucose tolerance test as factors associated with increased morbidity. Yamanaka et $\mathrm{al}^{7}$ reported age as a risk factor. Miyagawa et al. ${ }^{6}$ demonstrated, in a multivariate analysis, that operation duration, extension of resection and preoperative cardiovascular disease correlated with postoperative complication period. Surprisingly, this author observed, in a univariate model, that a histologically normal liver (except for the tumor) was 
associated with higher morbidity rates. This finding was possibly due to the more extensive liver resections performed in patients without underlying liver disease. In addition to this finding, we also observed that the size of tumor and blood transfusion were predictors of major postoperative complications.

In this study extension of liver resection was a predictor factor of complication; Miyagawa et al. ${ }^{6}$ found that extension of resection correlated with postoperative complication in a multivariate model. in this study, Major complications were observed with rate of $29.4 \%$ in patients who were subjected to major resections.

Liver resection in patients with cirrhosis presented a mortality rate of $58 \%$ by $1970 .{ }^{8}$ In the eighties, Asian and Western countries reported rates of $20 \%{ }^{9}$ Due to advances in perioperative management, refinement of surgical technique, reduced hospital stay and reduced requirement for intensive care unit monitoring, the mortality rates in the nineties reached a plateau near $10 \%$, Although our rate is zero mortality, compared to $8.4 \%$ of other centers, this difference may be due to a smaller number of patients in our study, zero-mortality rate has been reported by a few highly specialized centers.

Age was also described as a risk factor by Dimick et al, ${ }^{5}$ who found, in a recent multicentric study, that low-volume hospitals for hepatectomies, patients older than 65 years of age, major liver resection, primary hepatic malignancy as indication for resection and severity of hepatic dysfunction were all independent predictors of mortality.

In this study, the most common complication was liver decompensation accounting for $24.1 \%$, were observed in cirrhotic patients. (Due to high prevelance of cirrhosis in our cases because of prevelance of $\mathrm{HBV}$ in Egypt) Also Recurrence rate was $13.8 \%$, are often associated with focal infiltrated surgical margin and needed reintervention in 3.4\%.

Biliary complications represent another major topic in liver surgery. In a recent report, Tanaka et $\mathrm{al}^{9}$ published a leakage rate of $7.2 \%$, with a significant number of the patients with this complication requiring reintervention. In a series of $5.5 \%$ of biliary leakage, LAM et al., in agreement with BISMUTH et al, concluded that intraoperative dye tests significantly reduced fistula rates. ${ }^{9}$

In this study, however, leakage was not significant in most cases; we agree with others that the routine use of dye tests is not justified, since only a small percentage of patients need reintervention. In addition, even with routine assessment of leakage with this test, cases of biliary fistula are still observed.

In conclusion, our findings demonstrate that liver resection can be performed with low mortality and acceptable morbidity rates. Surgical complications can be reduced by employing meticulous surgical technique and, whenever indicated, vascular exclusion.

In addition, Use of vaccine against HBV eventually decreases the incidence, especially in endemic areas, and prevents the development of cirrhosis, which predispose to hepatocellular carcinoma.

\section{Reference}

1- Stone HH, Long WD, Smith RB $3^{\text {rd }}$, Haynes CD: Physiologic considerations in major hepatic resections. Am J Surg 1969; 117: 78-84.

2- Cai JQ, Hu JQ, Bi XY, Zhao JJ, Che X, Xie SL, et al: Long-term effect of united hepatectomy and splenectomy on treatment of hepatocellular carcinoma complicated with cirrhosis and hypersplenism. Chin Med $J$ 2004; 84: 6-8.

3- Beavers KL, Sandier RS, Shrestha R: Donor morbidity associated with right lobectomy for living donor liver transplantation to adult recipients: a systematic review. Liver Transpl 2002; 8: 110-117.

4- Virani S, Michaelson JS, Hutter MM, et al: Morbidity and mortality after liver resection: Results of the patient safety in surgery study. $J$ Am Coll Surg 2007; 204: 1284-1292.

5- Dimick JB, Cowan JA Jr, Knol JA, Upchurch GR Jr: Hepatic resection in the Unites States: indications, outcomes, and hospital procedural volumes from a nationally representative database. Arch Surg 2003; 138: 185-191.

6- Miyagawa S, Kawasaki S, Noike T, Nomura $\mathrm{K}$, Kobayashi A, Shimada R, Imamura H: Liver regeneration after extended right hemihepatectomy in patients with hilar or diffuse bile duct carcinoma. 1999; 46: 
364-368.

7- Yamanaka N, Okamoto E, Kawamura E, et al: Dynamics of normal and injured human liver regeneration after hepatectomy as assessed on the basis of computed tomography and liver function. Hepatology 1993; 18: 79-85.

8- Foster JH, Berman MM: Solid liver tumors.
Major Probl Clin Surg 1977; 22: 89-96.

9- Tanaka S, Hirohashi K, Tanaka H, Shuto T, Lee SH, Kubo S, Takemura S, YamamotoT, Uenishi T, Kinoshita $\mathrm{H}$ : Incidence and management of bile leakage after hepatic resection for malignant hepatic tumors. $J \mathrm{Am}$ Coll Surg 2002; 195: 484-489. 